\section{Vitamin B supplementation does not slow cognitive decline in Alzheimer disease}

High plasma homocysteine levels have been linked to raised levels of amyloid peptides and to cognitive decline in individuals with Alzheimer disease (AD). Aisen et al. have now established that although high-doses of $B$ vitamins reduce homocysteine levels in patients with mild to moderate $A D$, supplementation with these vitamins does not slow cognitive decline.

This multicenter trial enrolled individuals over 50 years of age with probable $A D$ and a Mini Mental State Examination score in the range 14-26. Patients were randomly assigned in a $3: 2$ ratio either to active treatment with $5 \mathrm{mg}$ folic acid (Vitamin $B_{9}$ ), $1 \mathrm{mg}$ vitamin $B_{12}$ and $25 \mathrm{mg}$ vitamin $B_{6}$ daily, or to placebo.

At 18-month follow-up (median 17.9 months), homocysteine levels had decreased significantly from baseline in patients receiving active treatment $(n=204 ; P<0.001)$ but had not changed markedly in individuals receiving placebo $(n=140)$. No difference was evident between the active treatment group and the placebo group in the rate of change on the cognitive subscale of the Alzheimer Disease Assessment Scale, nor in the rate of decline on the Clinical Dementia Rating sum of boxes test. The numbers of adverse events, hospitalizations and deaths were also similar in the two groups; however, the number of adverse events involving depression was significantly higher in the active treatment group $(P=0.02)$.

These results do not support the general recommendation for vitamin B supplementation in patients with mild to moderate $A D$ and normal vitamin levels.

Original article Aisen PS et al. for the Alzheimer Disease Cooperative Study (2008) High-dose B vitamin supplementation and cognitive decline in Alzheimer disease:

a randomized controlled trial. JAMA 300: 1774-1783

\section{Glutaminyl cyclase inhibition reduces Alzheimer disease pathology and symptoms}

Pyroglutamate $(\mathrm{pE})$-modified, N-terminaltruncated $A \beta$ peptides are thought to contribute to the development of Alzheimer disease (AD) by increasing the deposition of $A \beta$ peptides in neocortical brain structures. Schilling et al. have shown that inhibition of glutaminyl cyclase-an enzyme that catalyzes the in vitro formation of one such peptide, $A \beta_{3(\mathrm{pE})}-$ attenuates plaque formation and improves memory function in animal models of AD.

The glutaminyl cyclase inhibitor PBD150 was tested in 4-month-old and 6-month-old mice of the Tg2576 strain, which starts to develop amyloid plaques at 10-12 months of age. PBD150 produced dose-dependent decreases in $A \beta_{3(\mathrm{pE})-42}$ concentrations, which in turn lowered levels of other $A \beta$ peptides. Immunohistochemical testing of brain samples from these mice revealed significantly lower plaque burden in mice treated with PBD150 than in controls $(P<0.05)$. In addition, mice treated with high-dose PBD150 had better contextual memory than control mice and those treated with low-dose PBD150 $(P=0.0471)$. PBD150 also reduced $A \beta_{3(\mathrm{pE})-42}$ concentrations in 10month-old Tg2576 mice, which already have distinct plaque pathology, but the agent had no effect on concentrations of other amyloid peptides or on total plaque burden.

PBD150 produced similar effects on $A \beta_{3(\mathrm{pE})-42}$ concentrations, concentrations of other amyloid peptides, plaque burden, and memory function in TASD-41 mice-a transgenic mouse model of $A D-$ and reduced $A \beta_{3(\mathrm{pE})-42}$ concentrations in transgenic Drosophila with neuron-specific expression of $A \beta_{3(\mathrm{pE})}$ peptides.

The authors suggest that glutaminyl cyclase inhibition could provide a new therapeutic option in patients with $\mathrm{AD}$ or other amyloidoses.

Original article Schilling S et al. (2008) Glutaminyl cyclase inhibition attenuates pyroglutamate $A \beta$ and Alzheimer's disease-like pathology. Nat Med 14: 1106-1111

\section{Cortical neurons can directly control paralyzed muscle}

Recent studies have shown that a monkey can control a robotic arm connected directly to its brain, with the aid of a computer to decode neuronal signals. A new study by Moritz et al. has taken this method one step further to reroute neuronal control signals directly to a paralyzed muscle in two monkeys.

A brain-machine interface used motor cortex cells in areas corresponding to the hand and wrist to convert signals from single neurons into 